\title{
Maylis de Kerangal sur les grands chemins de Claude Simon
}

\section{(2) OpenEdition}

Journals

Édition électronique

URL : http://journals.openedition.org/ccs/1837

DOI : $10.4000 /$ ccs. 1837

ISSN : 2558-782X

Éditeur :

Presses universitaires de Rennes, Association des lecteurs de Claude Simon

\section{Édition imprimée}

Date de publication : 30 août 2018

Pagination : $211-213$

ISBN : 978-2-7535-7489-2

ISSN : $1774-9425$

Référence électronique

"Maylis de Kerangal sur les grands chemins de Claude Simon », Cahiers Claude Simon [En ligne], 13।

2018, mis en ligne le 30 août 2019, consulté le 24 septembre 2020. URL : http://

journals.openedition.org/ccs/1837 ; DOI : https://doi.org/10.4000/ccs.1837 


\section{Maylis de Kerangal sur les grands chemins de Claude Simon, Carnets de Chaminadour, $\mathrm{n}^{\circ} 11,2016,397 \mathrm{p}$.}

Depuis 2006, les rencontres de Chaminadour qu'organise Hugues Bachelot en complicité avec Pierre Michon, réunissent autour de deux écrivains chaque fois différents un public de passionnés de littérature. De ces deux écrivains, l'un orchestre les recherches et lectures sur l'œuvre de l'autre. En septembre 2015, c'est autour de Claude Simon que Maylis de Kerangal réunit un certain nombre de ses lecteurs, spécialistes ou non, écrivains ou non, pour des échanges à la fois savants et peu académiques, sérieux et décontractés.

Un gros volume, Maylis de Kerangal sur les grands chemins de Claude Simon, rend compte de ces échanges, en laissant entrevoir l'atmosphère souvent joyeuse qui les entourait dans un cahier photographique. Organisées par deux associations de lecteurs ${ }^{1}$, les rencontres s'adressent davantage à ceux-ci qu'à des chercheurs, et elles sont présentées d'entrée à des lycéens. Leur objectif est clairement d'inciter à la lecture. Pour cela, peu de communications "académiques " et beaucoup d'entretiens, de dialogues, de tables rondes: la transcription des propos, qui note les rires et les applaudissements, donne le ton, et prête sa voix au public.

L'œuvre de Simon est abordée par le biais de ses lectures, celles des écrivains présents, d'un créateur comme Dubuffet, d'éditeurs, de chercheurs, de traducteurs, et de médiateurs. Les études ont fonction propédeutique, facilitant au mieux l'entrée dans chacune des deux œuvres ou bien précisant un parcours de lecture, un processus d'édition ou de traduction. Les dialogues alternent avec les discours, selon des modalités variées. Ainsi nombre d'éléments de savoir et de commentaire savants passent-ils par des formes orales, plus "pédagogiques". Suivant le fil conducteur de la lecture et de tous les territoires qu'elle ouvre, sans en occulter les difficultés, le volume présente d'abord Maylis de Kerangal, dont l'univers, plus immédiat, donne accès au massif imposant de l'œuvre de Claude Simon. Puis d'autres voix la rejoignent pour dire leur rencontre avec les livres de Simon, et ses effets sur leur écriture, ou leur travail.

Un premier dialogue permet à Maylis de Kerangal et à Pierre Michon d'évoquer leur lecture de Simon et de donner l'orientation générale: il s'agit de désaffubler Simon de la fausse image d'auteur "cérébral " ou "formaliste ». Le lire est moins affaire de compréhension que de sensations, dit Maylis: de sa langue organique, de ses descriptions surgissent des visions, comme si la littérature était une sorte de sonde pour descendre dans les plis du temps et de l'Histoire. Mais ce n'est qu'à la seconde tentative qu'elle

1. L'Association des lecteurs de Marcel Jouhandeau et des amis de Chaminadour et l'Association des Lecteurs de Claude Simon. 
a pu se laisser couler dans le flux de sa prose. Rien de tel pour Pierre Michon, immédiatement happé par la force de son langage, tout en entendant continûment celui de Faulkner en arrière-plan. Maylis de Kerangal souligne le caractère "extrêmement vivant" (p. 112) des livres de Simon, alors que Michon les voit comme " un immense tombeau " (p. 115). De même, leur conception de l'influence diffère, l'un jugeant celle de Faulkner comme un amoindrissement de l'originalité de Simon, alors que l'autre considère son appropriation de l'œuvre simonienne comme un " épaulement » (p. 38).

Par delà les variations individuelles, un même souci de lire Simon sans a priori se retrouve dans les échanges entre les autres écrivains invités - dont beaucoup ont participé à une même revue, Inculte: expérience des sensations, du phrasé puissant et vertigineux, de la force du rythme, de l'ensevelissement-enfoncement (Marie-Hélène Lafon, p. 288), «des zooms sur les détails » qui font voir (Luc Lang, p. 289), de « l'obstination » de la phrase qui "organise la multiplication des mondes " (Arno Bertina, p. 294). Plusieurs racontent qu'ils sont entrés dans l'œuvre avec lenteur et tâtonnements, après d'autres lectures (Proust entre autres). Tous lisent pour écrire, écrivent parce qu'ils ont lu, et parlent d'une sorte d'autorisation, d'une formidable liberté donnée par Simon à leur écriture: à la prolifération interne de la phrase pour Christophe Pradeau, la construction de dispositifs pour Arno Bertina, la montée simultanée des lignes narratives pour Luc Lang, la possibilité de " décloisonner " les temps pour Marie-Hélène Lafon, la juxtaposition, superposition ou opposition des images pour Oliver Rohe (p. 337), la construction d'une sorte de « jeu de cubes " pour Mathias Énard (p. 339). Claro redéfinit l'influence comme une relation qui se construit pour reprendre sans répéter: " déjà, quand on lit, il faut construire le filtre, construire l'influence et après laisser rentrer des choses » (p. 341). Ces propos d'écrivains sont prolongés par les exemples d'absents remarquables (Jean-Paul Goux, Mathieu Larnaudie, Pierre Bergougnioux, Laurent Mauvignier...) dans la forte synthèse que propose Dominique Viart du « legs » de Claude Simon: essentiellement, cette " libération » déjà évoquée et une éthique paradoxale de l'écriture qui " conjugue une absolue lucidité sur son propre in-savoir et une dynamique herméneutique aussi insistante» (p. 323).

Les interventions de type universitaire, peu nombreuses, contribuent aux visées des rencontres en proposant des parcours de lecture. Deux études pionnières de l'œuvre de Maylis de Kerangal, celles de Cécile Yapaudjian-Labat et de Pierre Schoentjes, l'abordent par la question du lieu, du paysage: la première observe, dans les trois derniers romans, les mouvements, les voyages ou le passage d'un monde à l'autre, de la vie à la mort et inversement, soulignant l'énergie et la vitesse de la phrase qui cherche à tout embrasser. Le second s'interroge sur la sorte de lieu qui naît dans Naissance d'un pont, sur le rapport de celui-ci à la nature, aux enjeux de la modernité globalisée et de l'écologie. Pierre Schoentjes note que l'écriture épique de l'auteur valorise le mouvement technologique plus que les exigences écologiques. L'intervention à deux voix de Jean-Yves Laurichesse et Jean-Louis Tissier sur les lieux de mémoire de Simon est étayée par des illustrations, croquis et photographies de l'écrivain. Le littéraire et le géographe présentent ses lieux d'initiation historique et familiale: depuis les champs de bataille où est mort le père jusqu'à ceux de la guerre de 40 , paisibles et meurtriers, et aux images 
des voyages, comme la terre vue du ciel avec fascination. Le " parti pris des paysages " (p. 142) de Simon les lui fait décrire " comme si on ne les avait jamais vus ».

Dans sa "tentative de restitution d'un parcours de lecture", Alastair B. Duncan raconte son expérience de lecteur "noyé " et " exalté " (p. 165) par La Route des Flandres en 1966, puis celle d'un chercheur sur une œuvre en évolution constante. Il raconte ses échanges avec Simon, rencontré dès 1968, et la relation de confiance établie dans la distance et la discrétion, jusqu'au moment où il se voit confier la responsabilité de l'édition en Pléiade et la tâche délicate de concilier les exigences de l'éditeur et celles de l'auteur. C'est aussi un récit que fait Brigitte Ferrato-Combe des relations de Simon avec Dubuffet, celui d'un échange épistolaire et d'une admiration réciproque, fondée sur des affinités esthétiques: leur vision de la réalité et leur choix d'un retour au primordial et d'une "composition simultanée" (p. 375). David Zemmour, lui, ne raconte pas mais décrit la langue de Simon. À partir d'exemples précis, il explique pourquoi sa phrase s'écarte de la phrase canonique: par l'ordre des mots, les segments courts juxtaposés, le rythme, elle formule - au plus près - les sensations et, par l'évocation minutieuse de détails, cherche à communiquer l'intensité du regard et de l'émotion.

La richesse des entretiens autour de Simon tient aussi à la présence des médiateurs essentiels que sont l'éditeur, les traducteurs, les critiques, etc. Ainsi Hugues Pradier, directeur éditorial de la Bibliothèque de la Pléiade, lève un peu le voile sur la fabrique des deux volumes consacrés aux ouvres de Simon, signes et vecteurs de consécration, de "métamorphose ", et sur la réticence de Simon à livrer brouillons et secrets familiaux. Il revient aussi sur le "cas Faulkner " pour souligner que c'est grâce à la phrase libérée de Simon que Faulkner a pu être traduit plus fidèlement. Les traducteurs intervenants, Maria Baiocchi, Jufang Jin et Alexei Vishnyakov, avec Claro, traducteur écrivain, parlent de leurs expériences à l'intersection des langues et des cultures, entre adaptation et fidélité: travail sur le lexique et les registres de langue, sur le rythme et la ponctuation, sur les reprises simoniennes où Claro voit comme les traces d'un effort de traduction. Une dernière table ronde est l'occasion d'échanges entre bibliothécaire, éditeur, critique, directrice de la Maison des écrivains et libraires sur leur accompagnement de la réception des livres de Simon; ce sont autant d'éclairages sur la métamorphose qu'a connue l'œuvre de Simon, écrivain novateur méconnu (Marianne Alphant dit la surprise des Suédois de la voir seule représentante de la presse française à Stockholm en 1985) devenu grand auteur " classique " du $\mathrm{xx}^{\mathrm{e}}$ siècle.

Ainsi ces rencontres témoignent-elles de la complexité de ce qui entoure la lecture d'une œuvre exigeante: elle ne se développe que grâce à la réunion de facteurs et d'intercessions multiples. La lecture est certes un acte solitaire, mais où dialoguent et convergent nombre de voix lointaines ou proches mais silencieuses. En particulier quand cet écrivain est un pionnier qui introduit à des territoires encore inexplorés. 